\title{
- Que se Produz de Conhecimento Sobre Inova- ção? Uma Breve Análise das Características dos Artigos de Inovação Publicados nos Anais do EnANPAD (1 997-2009)
}

\author{
Giancarlo Gomes ${ }^{1}$ \\ Denise Del Pra Machado \\ Olivo Tiago Giotto ${ }^{3}$
}

\begin{abstract}
Resumo
Este estudo analisa a produção científica na área de inovação dos Encontros da Associação Nacional de Pós-Graduação e Pesquisa em Administração (EnANPAD). Em relação ao plano de pesquisa, este trabalho se caracteriza como exploratório, longitudinal, tendo como forma de pesquisa a bibliográfica. A pesquisa é do tipo desk research de 1997 até 2009. Em síntese, é possível perceber que FEA/USP e Universidade Federal do Rio de Janeiro se destacam na produção de artigos escritos em sua maioria por dois autores. Sendo um autor da COPPEAD/ UFRJ, com maior número de produções. A área com maior incidência de artigos é de Ciência, Tecnologia e Inovação. Dentre os artigos que fazem referência à metodologia empregada, predominam os artigos de natureza descritiva. O tipo de inovação em destaque é a tecnológica. A sugestão acerca da análise das metodologias pode direcionar estudos futuros, além da extensão da presente pesquisa, utilizando os mesmos critérios em outros eventos reconhecidos pela CAPES.
\end{abstract}

Palavras-chave: Inovação. Inovação tecnológica. Produção científica.

\footnotetext{
${ }^{1}$ Doutorando em Ciências Contábeis e Administração pela Universidade Regional de Blumenau. Endereço: PPGAd-Programa de Pós-Graduação em Administração PPGAd - campus I - sala D-102. Rua Antônio da Veiga, 140, CEP 89012-900 - Victor Konder Blumenau - SC. E-mail: giancarlo@pzo.com.br.

${ }^{2}$ Doutor em Administração de Empresas pela Fundação Getulio Vargas - SP (2004).Endereço: PPGAd - Programa de Pós-Graduação em Administração PPGAd - campus I - sala D-102. Rua Antônio da Veiga, 140, CEP 89012-900 - Victor Konder Blumenau - SC. E-mail: delpra@furb.br.

${ }^{3}$ Mestre em Administração pelo Programa de Mestrado em Administração da Fundação Universidade de Blumenau. Endereço: PPGAd - Programa de Pós-Graduação em Administração PPGAd - campus I - sala D-102 Rua Antônio da Veiga, 140 CEP 89012-900 - Victor Konder Blumenau -SC.E-mail: olivo@upf.br. Artigo recebido em: 25/05/2008. Aceito em: 05/03/2009. Membro do Corpo Editorial Científico responsável pelo processo editorial: Emerson Antônio Maccari.
} 


\section{Introdução}

As pesquisas que analisam a produção científica no âmbito acadêmico têm surgido visando uma verificação da qualidade científica brasileira. Dessa maneira, este artigo tem como objetivo principal analisar a produção científica da área inovação dos Anais do Encontro da Associação Nacional de Pós-Graduação e Pesquisa em Administração (EnANPAD) nos últimos 13 anos.

Percebe-se que a inovação tornou-se disciplina fundamental no cotidiano dos discursos e práticas de administração. Especialmente mediante ao contexto de competitividade crescente que o mundo dos negócios está submetido. Assim, empresas e demais organizações recorrem à inovação em busca de diferencial competitivo e diversificação.

A pesquisa em administração mostra-se sensível a essa problemática e revela a inovação como sendo um tema importante, pois nos últimos treze anos foi registrado um crescimento considerável da literatura sobre inovações, principalmente em inovações tecnológicas.

Dessa forma, os Anais do EnANPAD se estabelecem com um campo seguro e profícuo de pesquisas como a que este artigo se dedica, uma vez que, se trata de evento científico reconhecido e conceituado pela Coordenação de Aperfeiçoamento de Pessoal de Nível Superior (CAPES), como sendo Qualis A. Qualis é uma lista de divulgação de veículos para a produção intelectual, com base na avaliação de eventos e periódicos com circulações local, nacional e internacional, além de sua qualidade, pode ser enquadrada em A, B ou C. Essa classificação objetiva a avaliação do Sistema Nacional de Pós-Graduação stricto sensu (mestrado e doutorado) no Brasil (CAPES, 2009).

Após a introdução, o artigo apresentada fundamentação teórica e nela são abordados alguns conceitos sobre inovação e tipos de inovação, os quais auxiliaram na construção das categorias analíticas. Na terceira parte, discutese a metodologia empregada na criação das categorias analíticas utilizadas na investigação empírica. Posteriormente, os dados são analisados e, finalmente, na quinta e última etapa são apresentadas as considerações finais e sugestões para pesquisas futuras. 
O Que se Produz de Conhecimento Sobre Inovação? Uma Breve Análise das Características dos Artigos de Inovação...

\section{Plataforma Teórica}

\subsection{Inovação}

Existem muitas maneiras de definir inovação, das quais se destaca que inovação é qualquer ideia ou produto percebido pelo consumidor potencial como sendo novo (ENGEL; BLACKWELL; MINIARD, 2000). De acordo com Afuah (2003), a inovação está baseada no uso de um novo conhecimento tecnológico ou de mercado para oferecer um produto ou serviço novo aos clientes. Um produto pode ser considerado novo quando seu custo é baixo, seus atributos são melhorados ou inexistentes no mercado. Na mesma linha de pensamento, Zaltman, Duncan e Holbek (1973) versam que uma inovação pode ser definida como uma ideia, prática ou um bem material que é percebido como novo e de relevante aplicação.

A inovação deve ser compreendida como o processo que objetiva transformar as oportunidades em novas ideias e colocá-las amplamente em prática. O mesmo autor salienta ainda que a inovação é o ato ou efeito de inovar, ou seja, tornar algo novo; renovar; ou introduzir uma novidade. O termo eventualmente causa confusão, pois as pessoas tendem a entender inovação como invenção (TIDD; BESSANT; PAVITT, 2005).

O precursor na distinção dos conceitos de inovação e invenção foi Schumpeter (1939), em sua opinião e ao contrário de economistas anteriores, esses conceitos não devem ser confundidos. A distinção entre inovação $e$ invenção baseia-se no impacto econômico decorrente da introdução no mercado. As invenções, como descobertas científicas, podem permanecer muito tempo sem utilidade para o mercado, não afetando, assim, um sistema econômico. Uma inovação pode assumir várias formas e não necessariamente ser uma novidade na proporção de uma invenção, isto é, a inovação pode ocorrer por meio da aplicabilidade de uma ideia já existente a uma nova forma de operacionalizá-la ou a uma nova situação (SCHUMPETER, 1982).

A ligação entre inovação e desenvolvimento é salientada por Schumpeter $(1954$, p. 31) quando afirma que:

[...] o que nós, de forma não científica, chamamos de progresso econômico, significa, essencialmente, colocar recursos produtivos em usos ainda não tentados na prática $e$ 
retirá-los de utilizações para as quais servia, até o momento. A isto chamamos de inovação.

O progresso econômico acontece principalmente dirigido pelos avanços do conhecimento e aplicação da inovação, influenciando diretamente no desenvolvimento de nações. A Segunda Guerra Mundial foi o marco, especialmente para os EUA, na constatação de que a tecnologia impacta positivamente a economia. Essa constatação é que levou a Organização Europeia de Cooperação Econômica (OECE) a reunir dados para uma comparação que possibilitasse a padronização de ações em ciência e tecnologia, na ajuda de países menos desenvolvidos. Em 1963 é lançado o Manual Frascati que propõe um "Sistema Padrão para Avaliação em Pesquisa e Desenvolvimento" (OCDE, 1978, p. 16).

Na continuidade dos estudos da OECE, surge em 1992 a primeira versão do Manual de Oslo, que tem como objetivo básico servir de guia para a compilação de dados relativos à inovação tecnológica. Em 1996 é feita a última revisão desse manual, culminando com o reconhecimento de que as inovações consideradas "não-tecnológicas" também eram importantes. Tal reconhecimento veio dar cabo de uma distorção originária na década de 60: inovação só existe se for tecnológica. Assim, inovação passa a ser visualizada em seu sentido mais amplo, podendo também ocorrer no mercado, na organização, nos processos e nas técnicas.

Nesse mesmo sentido, Dosi (1988) argumenta que o processo de inovação pode ser entendido como a busca e descoberta, experimentação, desenvolvimento, imitação e adoção de novos produtos, novos processos de produção e novas formas organizacionais. Sendo complementado por Afuah (2003) que defende a ideia de que inovações, em qualquer âmbito, são as novas ideias que uma organização adota, independente de que já sejam adotadas em outras organizações. Ou seja, pode-se entender a inovação como a adoção de uma ideia já existente, mas que é nova para a organização que a está adotando. Nisso estão inclusos novos produtos, serviços, tecnologias, processos, procedimentos, sistemas ou arranjos sociais (DOSI, 1988; AFUAH, 2003; PENNINGS, 1998). É importante frisar que a inovação tem um tempo de vida e, após esse tempo, a empresa que obteve uma vantagem competitiva por meio de uma ação inovadora está sujeita a ser ultrapassada por concorrentes agressivos (PORTER, 1998). 
O Que se Produz de Conhecimento Sobre Inovação? Uma Breve Análise das Características dos Artigos de Inovação...

\subsection{Tipos de Inovação}

Os tipos de inovação mais relevantes, encontrados na literatura, são definidos como administrativa, tecnológica, produto, processo, radical $e$ incremental. Alguns autores agrupam-nas em administrativa e tecnológica, produto e processo, e, radical e incremental, para facilitar a descrição de uma tipologia (DAMANPOUR; EVAN, 1984; KIMBERLY; EVANISKO, 1981; DAFT, 1978; KNIGHT, 1967; DAMANPOUR, 1991).

Segundo Damanpour (1991), é fundamental que as organizações consigam diferenciar os tipos de inovação às quais estão propensas para adequar o comportamento organizacional e identificar os respectivos pontos fortes e fracos no seu desenvolvimento. Salienta ainda que os mais relevantes constantes na literatura são os seguintes pares: administrativa e tecnológica, produto e processo e radical e incremental.

O Manual de Oslo (OCDE, 2005, p. 25) identifica os tipos de inovação como: inovações de produto, inovações de processo, inovações organizacionais e inovações de marketing.

\footnotetext{
Uma empresa pode realizar vários tipos de mudanças em seus métodos de trabalho, seu uso de fatores de produção e os tipos de resultados que aumentam sua produtividade e/ou seu desempenho comercial. O Manual define quatro tipos de inovações que encerram um amplo conjunto de mudanças nas atividades das empresas: inovações de produto, inovações de processo, inovações organizacionais e inovações de marketing.
}

Conforme Daft (1978), inovações administrativas e tecnológicas culminam em processos diferentes de tomada de decisão por parte das organizações. A inovação tecnológica está relacionada basicamente ao processo de produção tecnológico de produtos e serviços. Como a inovação administrativa com a estrutura da organização e seus processos administrativos, ou seja, em uma dimensão gerencial (DAMANPOUR; EVAN, 1984; KIMBERLY; EVANISKO, 1981; KNIGHT, 1967).

A inovação de produto ou processo tem sido frequentemente utilizada pelas empresas com o objetivo de obtenção de diferenciais competitivos (ETTLIE, 1983; HULL, HAGE; AZUMI, 1985). Produtos inovadores são no- 
vos produtos ou serviços criados com o objetivo de atender necessidades encontradas no mercado, enquanto a inovação no processo é compreendida como os elementos que são agregados ao processo de operações, adição de materiais, tarefas, instrumentos ou fluxo de determinados mecanismos na produção de produtos ou serviços (KNIGHT, 1967; UTTERBACK; ABERNATHY, 1975). A diferenciação entre inovação de produto e processo parece ser a difundida pelos autores. Ao se considerar inovações em serviços, algumas diferenciações são propostas pelo Manual de Oslo:

Se a inovação envolve características novas ou substancialmente melhoradas do serviço oferecido aos consumidores, trate-se de uma inovação de produto;

Se a inovação envolve métodos, equipamentos e/ou habilidades para o desempenho do serviço novos ou substancialmente melhorados, então é uma inovação de processo;

Se a inovação envolve melhorias substanciais nas características do serviço oferecido e nos métodos, equipamentos e/ou habilidades usados para seu desempenho, ela é uma inovação tanto de produto como de processo (OCDE, 2005, p. 64).

Inovação de produto é entendida como a melhoria de bens ou serviços que serão utilizados por um mesmo grupo de consumidores. Porém, quando a melhoria de um produto resultar no alcance de um novo grupo de consumidores, ou seja, um grupo mais estratificado, refinado ou segmentado tratar-se-á de uma inovação de marketing. Ainda é possível que a inovação seja de produto e de marketing quando originar um novo conceito para o produto (OCDE, 2005, p. 65).

Essa distinção pode depender da natureza dos negócios da empresa. Um exemplo é a inovação referente a vendas pela internet. Para uma empresa que produz e vende bens, a primeira introdução do comércio eletrônico é uma inovação de marketing no posicionamento do produto. As empresas que estão em negócios de comércio eletrônico (por exemplo, empresas de "leilão", provedores de web sites que permitem que outras empresas anunciem ou vendam seus produtos, empresas que organizam a venda de bilhetes de viagem etc.) estão oferecendo "serviços de vendas". 
O Que se Produz de Conhecimento Sobre Inovação? Uma Breve Análise das Características dos Artigos de Inovação...

Para essas empresas, uma mudança significativa nas características ou nas capacidades de seu web site é uma inovação de produto (serviço).

O elemento básico para a diferenciação dos conceitos relativos a inovações em processos e organizacionais é de que as primeiras estão ligadas a novos equipamentos, softwares, técnicas ou procedimentos e as segundas se relacionam principalmente com as pessoas e com a organização do trabalho (OCDE, 2005).

Para Damanpour (1991), a diferença encontrada entre a inovação radical e incremental se refere à intensidade e extensão da mudança causada para o alcance da inovação. Dessa forma, a inovação radical será aquela cujas mudanças serão maiores e mais extensas se comparada com o estágio inicial. Do contrário ocorre uma inovação incremental, no qual as mudanças ocorrem a partir do incremento gradativo de inovações sequenciais.

\section{Metodologia}

Nesta etapa serão apresentados de forma detalhada os procedimentos realizados durante o processo da categorização das variáveis de medição, dos procedimentos de análise, da coleta de dados e as limitações da investigação.

\subsection{Critérios de Categorização e Mensuração das Variáveis}

O presente estudo analisou todos os artigos que continham a palavra "inovação" no título, resumo ou palavras-chave nos últimos 13 EnANPADs (1997-2009). Em relação ao plano de pesquisa, este trabalho se caracteriza como exploratório, longitudinal, tendo como forma de pesquisa a bibliográfica, enunciada por alguns autores como estudo de caso (GIL, 1987; VERGARA, 2004; HAIR JR. et al., 2003). Dessa forma, se desenvolveu uma pesquisa do tipo desk research por meio de um corte longitudinal em 13 anos (1997 até 2009) de EnANPADs.

Os critérios de categorização são elucidados nesta etapa, objetivando eliminar vieses de entendimento de como foram analisados os artigos. Este tópico teve como base o trabalho de Caldas, Tonelli, Lacombe (2002) e Vieira 
e Gava (2006) que também analisaram artigos das áreas de Recursos Humanos do EnANPAD (1991-2000) e de Marketing, publicados nos Anais do SEMEAD-FEA-USP (2000-2004). A seguir, serão apresentados os critérios utilizados na análise para, na continuidade, se expor a definição operacional e constitutiva de cada variável analisada.

Produção anual dos artigos: fez-se, nesta etapa, a verificação da quantidade de artigos produzidos com a palavra "inovação" constante no título, resumo e/ou palavras-chave. Foram encontrados, para o período de 1997 a 2009, 283 artigos, por meio de uma contagem de simples frequência nos Anais em meio digital (Compact Disk - CD). Verificou-se que o termo inovação é utilizado, mas no decorrer do artigo o conceito de inovação não é abordado, ou seja, se fala em "algo inovador", mas que não se utiliza nenhum conceito ou autor relacionado com inovação.

Classificação por tipo de inovação: após a pesquisa com a palavra "inovação" no título, resumo e/ou palavras-chave, na qual foram identificados 283 artigos, foi utilizado o critério de seleção por tipo de inovação. Nos 283 artigos se verificou a existência das palavras inovação tecnológica, produto, processo/produto, administrativa/tecnológica e radical/incremental em apenas 74 artigos, sendo isso o foco da pesquisa.

Universidades: para delineamento das Instituições de Ensino que mais publicaram sobre o tema inovação, foi verificada a procedência do autor, vinculando-o a uma universidade. Convém salientar que as obras publicadas em parceria entre autores de mais de uma universidade, levou-se em consideração as duas IES indicadas, ou seja, um artigo pode aparecer para mais de uma universidade.

Trabalhos com autores: com base em Vieira e Gava (2006), optouse pela classificação da quantidade de autores por artigo, analisando os trabalhos com um, dois, três ou mais autores.

Áreas: nesta etapa foi analisada a área (tema) do artigo publicado. Pelo fato de existirem subáreas dentro destas optou-se por se concentrar nas áreas principais, quais sejam: ACT (Administração de Ciência e Tecnologia); ADE (Administração Estratégica); ADI (Administração da Informação); AR (Administração Rural e Agroindustrial); EOR (Estudos Organizacionais); ESO (Estratégia em Organizações); GAG (Gestão de Agronegócios); GCT (Gestão da Ciência, Tecnologia e Inovação); GIN (Gestão Internacional); GOL (Gestão de Operações e Logística); GPG (Gestão Pública e Governança); GPR (Gestão de Pessoas e Relações de Trabalho); MKT (Marketing); ORG (Organiza- 
ções). Convém salientar que foram abordadas todas as áreas. Ao longo dos dez anos, a ANPAD modificou nomes; agrupou grandes áreas; e abriu outras, dessa forma se procurou fazer o levantamento em todas elas.

Autoria dos artigos: analisou-se, também, quais os autores que mais produziram na área de inovação, utilizado uma contagem de frequência simples.

Tipo de pesquisa: critérios propostos por Malhotra (2000) exploratória, descritiva e causal. Portanto, e segundo Malhotra (2000), pesquisa exploratória é aquela que prova a compreensão do problema enfrentado pelo pesquisador. A Pesquisa descritiva, por sua vez, descreve características ou funções do mercado (marcada pela formulação prévia de hipóteses específicas). $\mathrm{O}$ mesmo autor salienta ainda que a Pesquisa Causal determina relações de causa e efeito (manipulação de uma ou mais variáveis independentes).

\section{Análise dos Dados}

Essa seção está estruturada de acordo com os objetivos do estudo e de acordo com as variáveis mensuradas. A Tabela 1 apresenta a ocorrência de artigos com a ocorrência da palavra inovação no título, resumo e/ou palavras-chave nos EnANPADs de 1997 até 2009.

Convém salientar que foi observado uma diferença entre os dados constantes dos CDs e do site do EnANPAD nos anos de 2002 a 2009, nos anos de 2002, 2003, 2007, 2008 e 2009, o site apresenta dois, três e 19 artigos a mais, respectivamente, do que a quantidade constante nos CDs. A interpretação dos pesquisadores para essa diferença na quantidade apresentada e a existente nos CDs foi de que Coordenadores de Divisão podem enviar um "trabalho convidado para uma das Áreas da sua Divisão e até dois para as outras Divisões" (EnANPAD, 2009). Essa informação sugere que, nos cinco anos citados anteriormente, alguns coordenadores enviaram trabalhos na modalidade de "trabalho convidado".

Por outro lado, nos anos de 2004, 2005 e 2006 os CDs informam a existência de mais artigos que os relatados no site. Nos CDs as quantidades são maiores em sete, 28 e 17 artigos, respectivamente, para cada ano seguido. Esses dados sugerem que foram publicados mais artigos do que foram apresentados. Isso ocorre, muitas vezes, pelo autor enviar o artigo, fazer a inscrição, mas não comparecer durante a sessão. Dessa forma, para análise no presente trabalho, foram utilizadas as quantidades máximas para uma 
melhor configuração da relação entre artigos totais e os que continham a palavra inovação em sua formulação.

A Tabela 1 mostra a distribuição dos artigos nos últimos 13 anos. Apresentando o total geral de artigos publicados por ano de cada evento, os artigos que continham a palavra inovação no título, resumo e/ou palavras-chave $e$ os artigos que abordavam os tipos de inovação em estudo no presente artigo, ou seja, tecnológica, produto, processo/produto, administrativa/ tecnológica e radical/incremental.

Tabela 1: Distribuição dos artigos nos últimos 13 anos.

\begin{tabular}{|c|c|c|c|c|c|}
\hline \multicolumn{7}{|c|}{ Produção Anual de Artigos } \\
\hline Ano & $\begin{array}{c}\text { Total de } \\
\text { artigos }\end{array}$ & $\begin{array}{c}\text { Com a palavra } \\
\text { inovação }\end{array}$ & $\begin{array}{c}\text { \% com a palavra } \\
\text { inovação }\end{array}$ & $\begin{array}{c}\text { Estudos sobre } \\
\text { Inovação }\end{array}$ & $\begin{array}{c}\text { \% Estudos } \\
\text { sobre Inovação }\end{array}$ \\
\hline 1997 & 224 & 12 & 4 & 4 & 5 \\
\hline 1998 & 224 & 6 & 2 & 5 & 7 \\
\hline 1999 & 270 & 10 & 4 & 3 & 4 \\
\hline 2000 & 362 & 18 & 6 & 4 & 5 \\
\hline 2001 & 426 & 19 & 7 & 5 & 7 \\
\hline 2002 & 552 & 17 & 6 & 6 & 11 \\
\hline 2003 & 627 & 36 & 13 & 7 & 9 \\
\hline 2004 & 783 & 30 & 11 & 5 & 7 \\
\hline 2005 & 762 & 23 & 8 & 2 & 3 \\
\hline 2006 & 835 & 14 & 5 & 8 & 11 \\
\hline 2007 & 963 & 43 & 15 & 8 & 11 \\
\hline 2008 & 1.001 & 32 & 11 & 9 & 100 \\
\hline 2009 & 906 & 23 & 8 & 74 & \\
\hline TOTAL & 7.935 & 283 & 100 & & 7 \\
\hline
\end{tabular}

Fonte: Dados da pesquisa (2009).

Observa-se mediante análise da Tabela 1 que o ano de 2007 foi o que mais apresentou artigos com o foco de pesquisa em inovação e o ano de 1998 o menor percentual. No entanto, o ano de 2009 foi o que apresentou estudos sobre os tipos de inovação descritos para este artigo, observa-se também uma estabilidade nos últimos anos de pesquisa. A Associação Nacional de Pós-Graduação e Pesquisa em Administração, no ano de 2006, absorveu o XXIV Simpósio de Gestão da Inovação Tecnológica. Esse evento ocorre a cada dois anos, tendo em 2006 sua primeira versão participante do calendário de "seminários temáticos" da ANPAD (EnANPAD, 2009). A queda neste 
O Que se Produz de Conhecimento Sobre Inovação? Uma Breve Análise das Características dos Artigos de Inovação...

ano de artigos na área de inovação pode ter, no seminário temático, a explicação para o fenômeno. Como o seminário temático teve como tema principal "Inovação em Redes \& Redes de Inovação", acredita-se que muitos trabalhos foram inscritos neste seminário, deixando de comparecer no Encontro Anual.

O crescimento da produção com foco em inovação tem permanecido constante nos últimos 13 anos do EnANPAD. Com base no Gráfico 1, é possível visualizar que a inclinação da reta verde, porcentagem total de artigos, teve um aumento gradual nos 13 anos de pesquisa, no entanto, a reta azul, porcentagem dos artigos com a palavra inovação, não foi proporcional ao crescimento do próprio evento EnANPAD. A linha vermelha representa o percentual proporcional dos artigos com os tipos de inovação em pesquisa, nota-se que a reta se manteve constante nos anos em que foi realizado o estudo.

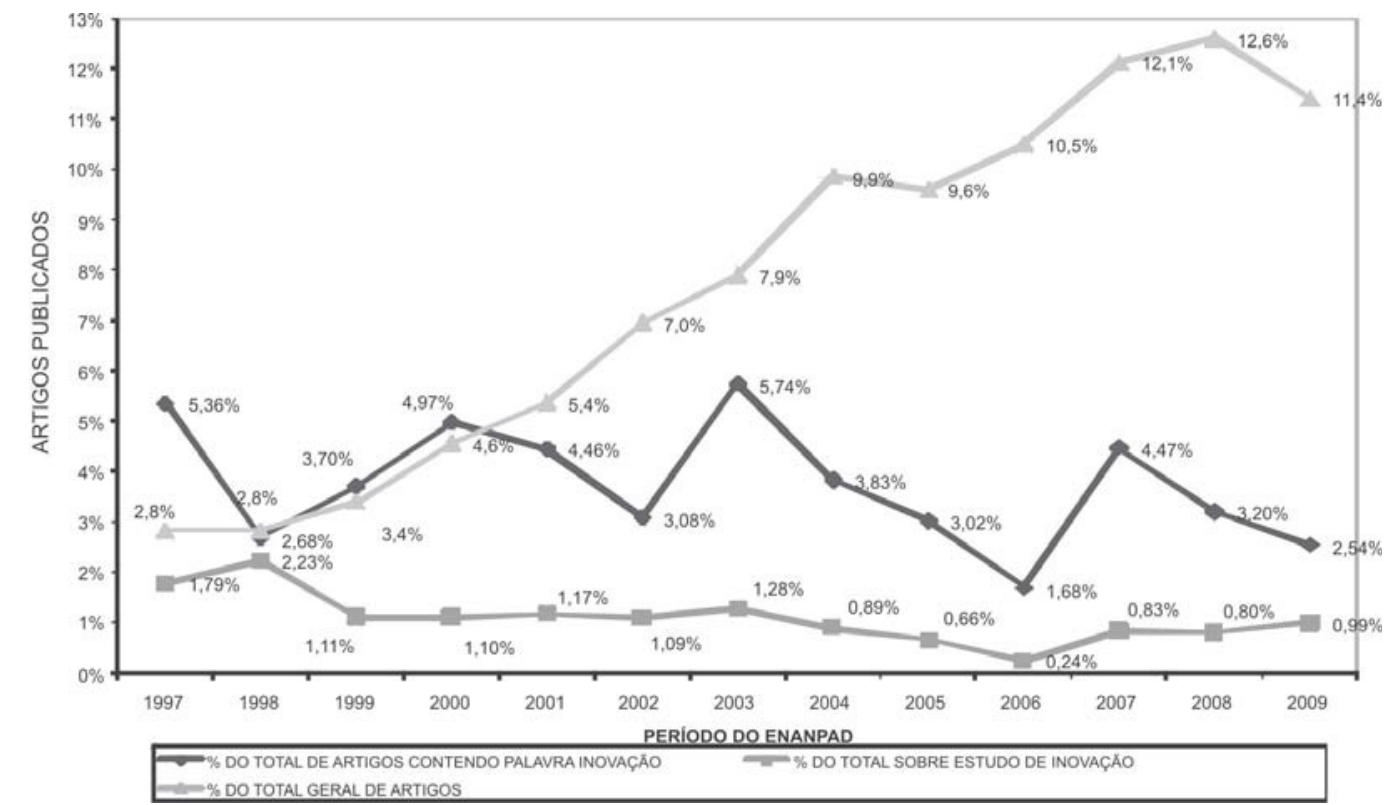

Gráfico 1: Linha temporal com a porcentagem da produção de artigos nos EnANPADs. Fonte: Dados da pesquisa (2009).

Na busca de classificar os artigos com a ocorrência da palavra inovação no título, resumo e/ou palavras-chave, buscou-se na etapa seguinte a ocorrência das palavras inovação tecnológica, produto, processo/produto, administrativa/tecnológica e radical/incremental. Conforme abordado na 
metodologia, convém reforçar que na análise dos artigos verificou-se que o termo inovação é utilizado, mas no decorrer do artigo o conceito de inovação não é abordado, ou seja, se fala somente em algo "algo inovador".

Nesse sentido, dos 283 artigos encontrados com a palavra inovação no título, resumo e/ou palavras-chave, apenas 74 fizeram parte das análises e os demais artigos foram desconsiderados, pois tratam apenas de estudos sobre inovação e não abordaram o tema de forma mais profunda. Assim, verificando a Tabela 2, percebe-se que a ocorrência de inovação tecnológica é muito superior que as demais inovações, atingindo o percentual de $81 \%$ das ocorrências. A inovação em produto surge em segundo lugar com $9 \%$ das ocorrências.

Tabela 2: Ocorrência dos tipos de inovação estudados.

\begin{tabular}{|l|c|c|}
\hline \multicolumn{1}{|c|}{ Tipo } & Ocorrência & \% \\
\hline Inovação Tecnológica & 60 & 81 \\
\hline Produto & 7 & 9 \\
\hline Processo/Produto & 2 & 3 \\
\hline Administrativa/Tecnológica & 3 & 4 \\
\hline Inovação Radical & 2 & 3 \\
\hline TOTAL & $\mathbf{7 4}$ & $\mathbf{1 0 0}$ \\
\hline
\end{tabular}

Fonte: Dados da pesquisa (2009).

Continuando a análise dos 74 artigos encontrados, considera-se importante o conhecimento das instituições de origem dos autores dos artigos encontrados. A Tabela 3 apresenta esse perfil. Constata-se que a Faculdade de Economia e Administração da Universidade de São Paulo - FEA/USP e a Universidade Federal do Rio de Janeiro destacam-se com nove artigos cada uma delas ao longo desses 13 anos. Ou seja, juntas chegam a quase um quarto de todos os artigos encontrados na pesquisa. A Escola de Administração e Economia da Fundação Getúlio Vargas - EAESP/FGV federais surge em segundo lugar com oito artigos. A Universidade Metodista Mackenzie apresenta seis artigos. Cinco artigos pesquisados no ano de 2005 não apresentaram a universidade na qual estavam vinculados os autores. As demais universidades apresentam de um a três artigos ao longo desses 13 anos. 
O Que se Produz de Conhecimento Sobre Inovação? Uma Breve Análise das Características dos Artigos de Inovação...

Tabela 3: Número de artigos por universidades ao longo dos 13 anos.

\begin{tabular}{|c|c|c|c|c|c|c|c|c|c|c|c|c|c|c|}
\hline \multirow{2}{*}{ Universidade } & \multicolumn{13}{|c|}{ Período EnANPAD } & \multirow{2}{*}{$\mathbf{T}$} \\
\hline & 97 & 98 & 99 & 00 & 01 & 02 & 03 & 04 & 05 & 06 & 07 & 08 & 09 & \\
\hline Universidade Federal do Rio de Janeiro & & & & & 1 & 2 & 2 & 2 & & & 1 & 1 & & 9 \\
\hline $\begin{array}{l}\text { Faculdade de Economia, Administração e } \\
\text { Contabilidade da USP }\end{array}$ & 1 & 1 & & & 1 & 1 & 1 & & & & 3 & 1 & & 9 \\
\hline Fundação Getúlio Vargas & & & & & 1 & 1 & 1 & 2 & & 1 & 1 & & & 8 \\
\hline Universidade Federal da Bahia & & 1 & 1 & 5 & & & & & & & & & & 7 \\
\hline Mackenzie & & & 1 & & & & 1 & & & & 3 & 1 & & 6 \\
\hline Universidade Federal de São Carlos & & & & & 1 & & & & & & 1 & & & 4 \\
\hline Universidade Federal de Minas Gerais & 1 & & & & & & & 1 & & & & & 1 & 3 \\
\hline Universidade Federal do Paraná & & & & & & 1 & & & & & & & & 3 \\
\hline Universidade Federal do Rio Grande do Sul & 1 & & & & & 1 & & 1 & & & & & 1 & 3 \\
\hline Universidade Federal de Lavras & 1 & 1 & & & & & & & & & & 1 & 1 & 3 \\
\hline Universidade de Brasília & & & & & & & & & & & & 1 & 2 & 3 \\
\hline Universidade Federal de Pernambuco & & & 1 & & & & & & & 1 & & & & 2 \\
\hline $\begin{array}{l}\text { Faculdade de Estudos Administrativos de Minas } \\
\text { Gerais FEAD-MG }\end{array}$ & & & & & & & & 1 & & & & & & 2 \\
\hline Pontifícia Universidade do Rio Grande do Sul & & & & & & 1 & & & & & & & 1 & 2 \\
\hline Universidade Federal de Santa Catarina & & & & 1 & & & & & & & & & 1 & 2 \\
\hline Universidade do Vale do Rio dos Sinos & & & & & & & 1 & & & & & & & 2 \\
\hline Instituto Superior de Economia e Gestão & & & & & & & & 1 & & 1 & & & & 2 \\
\hline Não Consta a Universidade & & & & & & & & & 5 & & & & & 5 \\
\hline TOTAL & & & & & & & & & & & & & & 88 \\
\hline
\end{tabular}

Fonte: Dados da pesquisa (2009).

A pesquisa teve ainda o intuito de identificar a quantidade de pesquisadores por artigo, assim de acordo com os dados contidos na Tabela 4, a maioria dos artigos pesquisados foi escrita por dois autores, num total de 45 artigos e atingindo aproximadamente $61 \%$ do total. Foram encontrados nove artigos escritos com um autor e nove artigos escritos com mais de três autores, atingindo $24 \%$ do total. Artigos escritos com três autores representam $15 \%$ dos artigos pesquisados.

Já os artigos escritos com até dois autores representam $45 \%$ dos artigos pesquisados, esta preferência predominante talvez se justifica pelo fato de que é possível trabalhos em conjunto do tipo orientador-orientado, parceria 
entre professor e aluno, trabalhos muitas vezes originários de dissertações de mestrado ou teses de doutorado.

Tabela 4: Quantidade de autores por artigos.

\begin{tabular}{|c|c|c|c|c|c|c|c|c|c|c|c|c|c|c|c|}
\hline \multirow{2}{*}{$\begin{array}{c}\mathrm{N}^{\circ} \text { de } \\
\text { autores }\end{array}$} & \multicolumn{13}{|c|}{ Período EnANPAD } & \multirow[b]{2}{*}{ Total } & \multirow{2}{*}{$\%$} \\
\hline & 1997 & 1998 & 81999 & 2000 & 2001 & 2002 & 2003 & 2004 & 42005 & 2006 & 2007 & 2008 & 2009 & & \\
\hline 1 Autor & 3 & 1 & 1 & 1 & & & 1 & 1 & & & 1 & & & 9 & 12 \\
\hline 2 Autores & & 2 & 3 & 5 & 4 & 8 & 5 & 4 & 2 & 1 & 3 & 5 & 3 & 45 & 61 \\
\hline 3 Autores & 1 & & & & & & 1 & 2 & 1 & 1 & 2 & & 3 & 11 & 15 \\
\hline Mais de 3 & & & & & & & 1 & & 2 & & 2 & 2 & 2 & 9 & 12 \\
\hline TOTAL & 4 & 3 & 4 & 6 & 4 & 8 & 8 & 7 & 5 & 2 & 8 & 7 & 8 & 74 & 100 \\
\hline
\end{tabular}

Fonte: Dados da pesquisa (2009).

Mediante a análise da autoria dos artigos selecionados, observa-se de acordo com os dados constantes na Tabela 5 que o autor com a maior incidência é César G. Neto (COPPEAD/UFRJ) que foi autor de quatro artigos (2002, 2004, 2007 e 2009). Em segundo lugar, aparece Sérgio Lex (MACKENZIE) com três artigos (2007 e 2009). Os demais autores apresentam dois artigos como sendo de sua autoria. De acordo com a Tabela 5, constata-se que o maior intervalo encontrado entre um artigo e outro é de cinco anos.

Tabela 5: Principais autores.

\begin{tabular}{|l|c|c|c|c|c|c|c|c|c|c|c|c|c|c|}
\hline \multirow{2}{*}{ Autores } & \multicolumn{8}{|c|}{ Publicaçóes de autores por anos de pesquisa } \\
\cline { 2 - 12 } & $\mathbf{9 7}$ & $\mathbf{9 8}$ & $\mathbf{9 9}$ & $\mathbf{0 0}$ & $\mathbf{0 1}$ & $\mathbf{0 2}$ & $\mathbf{0 3}$ & $\mathbf{0 4}$ & $\mathbf{0 5}$ & $\mathbf{0 6}$ & $\mathbf{0 7}$ & $\mathbf{0 8}$ & $\mathbf{0 9}$ & T \\
\hline César G. Neto & & & & & & 1 & & 1 & & & 1 & & 1 & 4 \\
\hline Sérgio Lex & 1 & & & & & 1 & & & & & & & & 2 \\
\hline Jaime E. Fensterseifer & 1 & & & & & 1 & & & & & & & & 2 \\
\hline Hubert Drouvot & & 1 & 1 & & & & & & & & & & & 2 \\
\hline Francisco L.C. Teixeira & & & & 2 & & & & & & & & & & 2 \\
\hline Rogério H. Quintella & & & & & & 1 & & 1 & & & & & & 2 \\
\hline Pierre Ohayon & & & & & & & & 1 & & & 1 & & & 2 \\
\hline Mônica Esteves Rodrigues & & & & & & 1 & & & 1 & & & & & 2 \\
\hline Silvio Antônio Ferraz Cario & & & & & & & 1 & 1 & & & & & & 2 \\
\hline Deborah Moraes Zouain & & & & & & & & & & & & & \\
\hline
\end{tabular}

Fonte: Dados da pesquisa (2009). 
O Que se Produz de Conhecimento Sobre Inovação? Uma Breve Análise das Características dos Artigos de Inovação...

Tabela 5: Principais autores.

\begin{tabular}{|l|c|c|c|c|c|c|c|c|c|c|c|c|c|c|}
\hline \multirow{2}{*}{ Autores } & \multicolumn{8}{|c|}{ Publicaçóes de autores por anos de pesquisa } \\
\cline { 2 - 12 } & $\mathbf{9 7}$ & $\mathbf{9 8}$ & $\mathbf{9 9}$ & $\mathbf{0 0}$ & $\mathbf{0 1}$ & $\mathbf{0 2}$ & $\mathbf{0 3}$ & $\mathbf{0 4}$ & $\mathbf{0 5}$ & $\mathbf{0 6}$ & $\mathbf{0 7}$ & $\mathbf{0 8}$ & $\mathbf{0 9}$ & $\mathbf{T}$ \\
\hline Maira Petrini & & & & & & & & & & & & & & \\
\hline Marlei Pozzebon & & & & & & & & 1 & & 1 & & & & 2 \\
\hline Maria Tereza Freitas & & & & & & & & 1 & & 1 & & & & 2 \\
\hline Moisés Ari Zilber & & & & & & & & & & & 2 & & & 2 \\
\hline Reynaldo C. Marcondes & & & & & & & 1 & & & & 1 & & & 2 \\
\hline Eduardo Raupp de Vargas & & & & & & & & & & & & & 2 & 2 \\
\hline Dany Flávio Tonelli & & & & & & & & & & & & 1 & 1 & 2 \\
\hline André Luiz Zambalde & & & & & & & & & & & & 1 & 1 & 2 \\
\hline Mônica Esteves Rodrigues & & & & & & & & & & & & 1 & 1 & 2 \\
\hline TOTAL & $\mathbf{2}$ & $\mathbf{1}$ & $\mathbf{1}$ & $\mathbf{2}$ & & $\mathbf{5}$ & $\mathbf{2}$ & $\mathbf{7}$ & $\mathbf{1}$ & $\mathbf{3}$ & $\mathbf{7}$ & $\mathbf{3}$ & $\mathbf{7}$ & $\mathbf{4 1}$ \\
\hline
\end{tabular}

Fonte: Dados da pesquisa (2009).

Na análise da distribuição dos artigos selecionados, de acordo com os dados apresentados na Tabela 6, observa-se que no ano de 1998 foi criada a área de Administração da Ciência e Tecnologia. Essa área existiu até o ano de 2004 e foi responsável por 39\% dos artigos encontrados. Constata-se, ainda, que no ano de 2005 foi criada a área de Gestão de Ciência, Tecnologia e Inovação, contendo $42 \%$ dos artigos pesquisados. Somando esses dois percentuais, chega-se a $81 \%$ dos artigos. É possível concluir que essas são as principais áreas que contemplam estudos de inovação.

Tabela 6: Distribuição dos artigos nas áreas de classificação do EnANPAD.

\begin{tabular}{|l|c|c|}
\multicolumn{1}{|c|}{ Área } & $\begin{array}{c}\text { No de } \\
\text { Ocorrência }\end{array}$ & $\mathbf{\%}$ \\
\hline ACT - Administração de Ciência e Tecnologia (1998 até 2004) & 29 & 39 \\
\hline ADI - Administração da Informação & 5 & 7 \\
\hline ESO - Estratégia em Organizações & 3 & 4 \\
\hline GCT - Gestão de Ciência e Tecnologia e Inovação (2005 em diante) & 31 & 42 \\
\hline MKT - Marketing & 3 & 4 \\
\hline ORG; AR; GIN; EOR; GPG; EOR; GAG; GOL; ADE; GPR; EPQ; APS & 3 & 4 \\
TOTAL & $\mathbf{7 4}$ & $\mathbf{1 0 0}$ \\
\hline
\end{tabular}

Fonte: Dados da pesquisa (2009). 
No que se refere ao tipo de pesquisa dos artigos selecionados, observase de acordo com os dados contidos na Tabela 7 que o número mais expressivo de tipo de pesquisa é o ausente, correspondendo a 39 artigos.

Esse é um número bastante elevado e pode indicar um desconhecimento ou descaso para o assunto por parte dos autores. Porém, é possível observar que o tipo de pesquisa descritiva conta com a preferência de $25,4 \%$ dos autores contra 15,3\% de pesquisas do tipo exploratória. Percebe-se que os pesquisadores estão mais empenhados em descrever os fenômenos referentes à inovação do que explorar novos âmbitos de pesquisa. Geralmente, as pesquisas descritivas se utilizam de ferramentas estatísticas para comprovação de hipóteses.

No entanto, a ausência do tipo de pesquisa causal, a qual procura estabelecer o entendimento na dimensão causa-efeito é uma metodologia ainda não adotada pelos pesquisadores da área.

Tabela 7: Distribuição dos artigos de acordo com o tipo de pesquisa.

\begin{tabular}{|l|c|c|c|c|c|c|c|c|c|c|c|c|c|c|}
\hline \multirow{2}{*}{ Tipo de pesquisa } & \multicolumn{10}{|c|}{ Período EnANPAD } \\
\cline { 2 - 17 } & $\mathbf{9 7}$ & $\mathbf{9 8}$ & $\mathbf{9 9}$ & $\mathbf{0 0}$ & $\mathbf{0 1}$ & $\mathbf{0 2}$ & $\mathbf{0 3}$ & $\mathbf{0 4}$ & $\mathbf{0 5}$ & $\mathbf{0 6}$ & $\mathbf{0 7}$ & $\mathbf{0 8}$ & $\mathbf{0 9}$ & \\
\hline Ausente & 1 & 3 & 1 & 5 & 4 & 4 & 3 & 4 & 2 & 1 & 4 & 2 & 5 & 39 \\
\hline Exploratória & 1 & & & & & 1 & 3 & 2 & & 1 & 1 & 3 & 1 & 13 \\
\hline Descritiva & 2 & & 3 & & & 3 & & 1 & 3 & & 3 & & 2 & 17 \\
\hline Exploratória/Descritiva & & & & 1 & & & 2 & & & & & 2 & & 5 \\
\hline Causal & & & & & & & & & & & & & & \\
TOTAL & $\mathbf{4}$ & $\mathbf{3}$ & $\mathbf{4}$ & $\mathbf{6}$ & $\mathbf{4}$ & $\mathbf{8}$ & $\mathbf{8}$ & $\mathbf{7}$ & $\mathbf{5}$ & $\mathbf{2}$ & $\mathbf{8}$ & $\mathbf{7}$ & $\mathbf{8}$ & $\mathbf{7 4}$ \\
\hline
\end{tabular}

Fonte: Dados da pesquisa (2009).

Em síntese, percebe-se que a FEA/USP, a UFRJ e a FGV se destacam na produção de artigos, sendo estes escritos, em sua maioria, por dois autores e tendo como autor com maior número de produções César Gonçalves Neto (COPPEAD/UFRJ). A área que contempla a maior incidência de artigos é a área de Ciência, Tecnologia e Inovação. Dentre os artigos que fazem referência à metodologia empregada, predominam os artigos de natureza descritiva. O tipo de inovação de maior destaque é a tecnológica. 
O Que se Produz de Conhecimento Sobre Inovação? Uma Breve Análise das Características dos Artigos de Inovação...

\section{Considerações Finais}

Levando em consideração a importância das inovações para a economia nacional, estudos sobre o tema tornaram-se relevantes. Nesse cenário, avaliar a produção científica na área de inovação tem o papel de revelar como estão as pesquisas referentes a esse tema nos últimos anos. No caso das inovações tecnológicas, pode-se observar que os estudos na área vêm ocupando a agenda dos pesquisadores brasileiros. Isso pode ser constatado ao se realizar o levantamento dos mais recentes artigos científicos na área apresentados nos últimos 13 anos do EnANPAD.

Realizados os passos apresentados na metodologia, pode-se constatar que o tema inovação não tem aumentado acima do crescimento normal do EnANPAD. Os percentuais se situam proporcionalmente constantes. Esse fato pode ser justificado pelo surgimento de eventos na área de ciência e tecnologia como o Seminário Temático ocorrido em 2006 em Gramado/RS, evento amparado pela ANPAD e com foco específico em Inovação. A clarificação dos critérios de avaliação de eventos, feito pela CAPES e publicado com mais ênfase a partir do ano de 2006 também pode vir a contribuir para o desenvolvimento da área de inovação em eventos específicos dessa temática.

Outros estudos necessitam ser feitos para abordagem de tema tão amplo e diversificado como inovação. Os artigos podem ser avaliados não só quanto à metodologia adotada, mas também na profundidade do uso das ferramentas de análise e no tamanho das amostras utilizadas. Sabe-se que aspectos de análise como inovação tecnológica e de produto são mais visíveis e mais facilmente mensuráveis, ficando a inovação em gestão relegada a metodologias qualitativas, muitas vezes não aceitas em algumas áreas do conhecimento.

A sugestão acerca da análise das metodologias pode direcionar estudos futuros, além da extensão da presente pesquisa, utilizando os mesmos critérios, em outros eventos reconhecidos pela CAPES. Essa dinâmica poderá ampliar a visão do assunto inovação, além de indicar caminhos na pesquisa científica sobre o assunto. 


\title{
What if Produz of Knowledge About Innovation? A Short Analysis of Features of Articles of Innovation Published in the EnANPAD Records (1997-2009)
}

\begin{abstract}
This article examines the scientific production in the area of innovation Encounters of the National Association of Post-Graduate Studies and Research in Administration (EnANPAD). The research in this case is characterized as exploratory, longitudinal, and making as a way to search the literature. The search is the type desk research from 1997 to 2009. In summary, realizes that it FEA/USP and University of Rio de Janeiro, it is in the production of articles, these writings, mostly by two authors, having the author of COPPEAD/UFRJ as the highest number of productions. The area with the highest articles incidences are Science, Technology and Innovation. Among the articles that refer to the methodology employed, dominate the articles of descriptive nature. The type of innovation is the emphasis on technology. The suggestion about the analysis of methodologies can conduct future studies, beyond the scope of this search, using the same criteria in other events recognized by CAPES.
\end{abstract}

Key-words: Innovation. Technology innovation. Scientific Production.

\section{Referências}

AFUAH, A. Innovation mangement: strategies, implementation and profits. New York: Oxford University Press, 2003.

CALDAS, Miguel; TONELLI, Maria; LACOMBE, B. Espelho, espelho meu: Meta-estudo da Produção científica em Recursos Humanos nos ENANPADs da década de 90. In: XXVI, Encontro da Associação Nacional de Programas de Pós-Graduação em Administração (EnANPAD). Salvador, Anais... Salvador: BA, 2002.

CAPES. Coordenação de Aperfeiçoamento de Pessoal de Nível Superior. Disponível em: <http://qualis.capes.gov.br/webqualis/>. Acesso em: $1^{\circ}$ nov. 2009. 
O Que se Produz de Conhecimento Sobre Inovação? Uma Breve Análise das Características dos Artigos de Inovação..

DAFT, R. L. A dual-core modelo f organizational innovation. Academy of Management Journal, Mississipi, v. 21, n. 2, p. 193-210, jun. 1978.

DAMANPOUR, F. Organizational innovation: a meta analysis of effects of determinants and moderators. Management Journal, New Jersey, v. 34, n. 3 555-590, 1991.

DAMANPOUR, F; EVAN, W. M. Organizational innovaion and performance: the problem of organizational lag. Administrative Science Quarterly, 29: 392-409, 1984.

DOSI, G. et al. Technical change and economic theory. London: Pinter Publishers, 1988.

EnANPAD. Encontros da Associação Nacional de Pós-Graduação e Pesquisa em Administração. Disponível em: <http://www.anpad.org.br/ eventos.php>. Acesso em: 2 abr. 2009.

ENGEL, J. F.; BLACKWELL, R. D.; MINIARD, P. W. Comportamento do consumidor. 8. ed. Rio de Janeiro: LTC, 2000.

ETTLIE, J.E. Organizational policy and innovation among suppliers to the food processing sector. Academy of Management Journal, Mississipi, v. 26 n.1, 27-44p., 1983.

GIL, Antônio Carlos. Métodos e técnicas de pesquisa social. São Paulo: Atlas, 1987.

HAIR JR., J. et al. Fundamentos de métodos de pesquisa em administração. Porto Alegre: Artmed Editora S.A., 2003.

HULL, F. M.; HAGE, J.; AZUMI, K. R\&D Management strategies: Americam versus Japan. IEEE Transactions on Enginnering Manegement, v. 32, p. 78-83, 1985.

KIMBERLY, J. R.; EVANISKO, M. J. Organizational innovation: the influence of individual organizational, and contextual factors on hospital adoption of technological and administrative innovations. Academy of Management Journal, Mississipi, v. 24, n. 4, p. 689- 713, dez. 1981.

KNIGHT, K. E. A descriptive model of intra-firm innovation process. Journal of Business, Chicago, v. 40, p. 478-496, 1967. 
Giancarlo Gomes - Denise Del Pra Machado • Olivo Tiago Giotto

MALHOTRA, Naresh. Pesquisa de Marketing: uma orientação aplicada. Porto Alegre: Bookman, 2000.

OCDE - Organização para a Cooperação e o Desenvolvimento. Manual Frascati: medição de atividades científicas e tecnológicas. João Pessoa: CNPq - IBICT, 1978. 150 p. (Cadernos de Informação em Ciência e Tecnologia, n. 2).

Manual de Oslo. Finep - tradução português, 2005.

PENNINGS, J. M. Innovations as precursors of organizational performance. In:

Information technology and organizational transformation innovation for the 21 st century organization. Edited by Robert d. Galliers e Walter R. J. Baets. Weinheim, Editora Wiley, 1998.

PORTER, Michael E. Criando as vantagens de amanhã. In: GIBSON, Rowan. Repensando o Futuro. São Paulo: Mackron Books, 1998.

SCHUMPETER, J. Business cycles. New York: McGraw Hill Books, 1939.

History of economic analysis. New York, NY, EUA: Oxford University press, 1954.

A teoria do desenvolvimento econômico. 3. ed. São Paulo: Abril Cultural, 1982.

TIDD, J.; BESSANT, J.; PAVITT, K. Managing innovation: integrating technological,market and organizational change. West Sussex, UK: John Wiley \& Sons, 2005.

UTTERBACK, J. M.; ALBERNATHY, W. J. A dynamic modelo of process and reorientation. Administrative Science Quarterly, 16: 203-215, 1975.

VERGARA, Sylvia Constant. Projetos e relatórios de pesquisa em administração. 5. ed. São Paulo: Atlas, 2004, 96p.

VIEIRA, V. A.; GAVA, R. Uma análise do conteúdo dos artigos de marketing publicados nos anais do SEMEAD-FEA-USP. In: IX SEMEAD - Seminários em Administração FEA/USP, 2006. São Paulo. Anais... FEA/USP, 2006.

ZALTMAN, G.; DUNCAN, R.; HOLBEK, J. Innovations and Organizations. New York: Wiley, 1973. 